\title{
POLÍTICA INDÍGENA EN COTOPAXI. \\ La demanda, la protesta y la participación del MICC'
}

José J. Egas ${ }^{2}$

\section{Resumen}

El siguiente artículo busca un análisis histórico del movimiento indígena y Campesino de Cotopaxi (MICC), ubicando tres momentos relevantes en la retrospectiva: en primer lugar, las luchas agrarias y la reivindicación de los derechos donde se considera los antecedentes a esta dinámica. Un segundo momento, plantea que el MICC atraviesa por una etapa caracterizada por las acciones de protesta enmarcadas dentro de los múltiples levantamientos y la acción colectiva generados a partir de su ubicación en la escena nacional con el levantamiento de 1990. Finalmente, se sugiere una tercera etapa, que estaría marcada por la participación en las elecciones y la gestión política interna a través de los cargos obtenidos donde se establecen ciertas lógicas de acción más apegadas al sistema democrático, que de una u otra forma resquebrajan la lógica interna de manejo político secularizando las prácticas de poder entre lo tradicional y lo moderno. En cada uno de los momentos se ilustra el contexto con ciertos ejemplos.

\begin{abstract}
The present article analyzes the political process of the Indigenous and Campesino Movement of Cotopaxi (MICC in Spanish) since the beginning of the 80's. The analysis of the principle events is deepened keeping in mind three political moments key to the dynamic of the movement. The first events of organization and establishment of the MICC were framed as the demand of rights. In second place keep in mind the rise and consolidation of the group as a new political stakeholder starting with the uprising of 1990 when a new phase of political protest began. Finally we analyze the incursion of the Movement in the electoral competitions, the pros and cons of the functioning of the "democratic" logic in the political consolidation of the indigenous movement at a provincial level. The present article, therefore, is an approximation towards the MICC from their politics taking into account the actors that intervene.
\end{abstract}

T a provincia de Cotopaxi, ubicada en la zona central de la sierra ecuatoriana es una de las provincias con mayor demografía étnica, se estima que el $39 \%$ de la población provincial puede ser categorizada como indígena (Ospina, 2006:22). Sin embargo, más allá de los datos cuantitativos, la provincia de Cotopaxi alberga una larga tradición de organización indígena y campesina cuya manifestación más clara han

1 El presente artículo está basado en una síntesis de la tesis de licenciatura realizada para el departamento de antropología bajo el título: "El movimiento indígena ecuatoriano. El caso del movimiento indígena y campesino de Cotopaxi reivindicación, protesta y participación".

2 Licenciado en Antropología PUCE. Maestría en Comunicación UASB. 
sido las diversas luchas desde mediados del siglo pasado así como las movilizaciones y levantamientos de los años recientes. El Movimiento Indígena y Campesino de Cotopaxi (MICC) ha sido la organización campesino indígena que ha constituido el principal espacio de reproducción de la práctica política indígena en la provincia.

\section{La demanda, inicios políticos del MICC: actores y escenarios.}

Las expresiones iniciales del MICC, concentrando inicialmente las expectativas del movimiento provincial, se ven reflejadas en los reclamos de las luchas contra el sistema hacendatario, ubicando el epicentro del conflicto en la irregular tenencia de la tierra y las desiguales formas de producción; así, las primeras luchas encuentran un adversario definido: el terrateniente y su institución: la hacienda. De tal forma, los primeros logros del MICC como organización estarán centrados en la recuperación y la adjudicación de tierras a ciertas comunidades; lo cual se constituirá en un segundo logro materializado en la presión hacia los hacendados para que vendan sus tierras a precios convenientes a los indígenas y campesinos, agilitando, de esta manera, el proceso de conformación y recuperación de los territorios comunales.

No menos importantes que las demandas de reivindicación de tierras fueron las exigencias por la implementación efectiva de una educación intercultural, en cuyo personal se incluya docentes indígenas que garanticen contenidos pedagógicos adaptados a la realidad local. En este sentido, los logros obtenidos por el movimiento indígena han sido influidos por una diversidad de actores externos. Cuatro han sido las modalidades de educación intercultural que han sido implementadas en la provincia desde fines de los años setenta.

La más importante de estas iniciativas y la que mayor duración ha mantenido en el área ha sido el Sistema de Escuelas Indígenas de Cotopaxi (SEIC) que:

“...ha desarrollado una experiencia educativa al servicio de la población indígena quichua hablante de las zonas de Chugchilán, Zumbahua, el Bajío (Saquisilí, Pujilí, Poaló) y Panyatuc; en las primeras desde 1976; y en la última desde 1988 [...] que se identifican como escuelas indígenas porque el proceso educativo es eminentemente antropocéntrico en cuanto ha confluido en el fortalecimiento de la identidad cultural [...] de los comuneros quienes son los educandos y a la vez los dueños de su propia educación” (Martínez y Burbano, 1994: 11).

La segunda de estas iniciativas de educación intercultural es la del Proyecto de Educación Bilingüe Intercultural (PEBI), que se empieza a desarrollar a partir de una investigación sociolingüística y educativa que se llevó a cabo entre 1981 y 1982. Para 1985 se suscribe un convenio entre el Ministerio de Educación y el Gobierno de Alemania Federal a través de GTZ (Deutsche Gesellschaft für Technische zusammenarbeit). Este proyecto se constituye la base de la colaboración de organizaciones quichuas locales y regionales. El proyecto EBI ha asentado su trabajo básicamente en cuatro áreas: investigación didáctica y lingüística, entrenamiento y formación de educadores bilingües, producción de material didáctico y difusión de la lengua y la cultura quichua (Krainer, 1996: 47). Este programa ha tenido una influencia prolongada en las áreas rurales de la sierra ecuatoriana; Cotopaxi 
ha participado en ciertos sectores de la zona andina a través de la instalación y puesta en marcha de escuelas interculturales.

La tercera forma de educación intercultural es la que se propicia a partir de la fundación de la Dirección Nacional de Educación Indígena Intercultural Bilingüe (DINEIIB), mediante la cual las escuelas con una educación hispano-parlante, básicamente las ubicadas en las zonas rurales, pasan a formar parte de la administración de la DINEIIB con sus delegaciones provinciales; esta dinámica se produce en 1989 (Martínez y Burbano, 1994: 11).

La cuarta experiencia de educación intercultural que tuvo cierto influjo en la provincia de Cotopaxi y en el resto de la sierra es el Centro de Investigación para la Educación Indígena (CIEI). Para 1978 se firmó un convenio entre el Ministerio de Educación y la Pontificia Universidad Católica del Ecuador (PUCE), implementándose la fase experimental de alfabetización quichua - castellano. Para los años entre 1980 y 1984 el proyecto se extiende a todo el país en los niveles de alfabetización, post-alfabetización, y educación bilingüe, intercultural, infantil.

Los propósitos pedagógicos impulsados por los sistemas de educación indígena en la provincia de Cotopaxi, especialmente los del SEIC por su larga presencia en la zona, han constituido además de logros académicos y formativos un fuerte apoyo institucional a la capacidad organizativa del MICC. Las acciones dentro de los programas mismos de enseñanza indígena en las escuelas rurales funcionaron en los primeros momentos de organización del MICC como catalizadores de las reivindicaciones y luchas del movimiento. Una muestra de estas constantes se ilustra en el gran número de dirigentes que participaron e incluso participan hasta la actualidad en las dinámicas de la educación y han ocupado u ocupan puestos en las instancias del MICC o de sus OSGs (Tibán, 2003).

\section{La influencia de la Iglesia}

En la década del 70, la Iglesia había sido la única institución presente en la zona del Quilotoa que además de iniciar el proceso de alfabetización y refuerzo del quichua y las experiencias pedagógicas interculturales, también fue una institución que administró proyectos de desarrollo importantes en la zona (el caso de FODERUMA), a través de los cuales se adquirió varios de los servicios básicos con los que cuenta el área de Zumbahua (Weismantel, 1994: 118).

En algunos casos la cuestión del restablecimiento económico y político en el mundo indígena adquirió mayor significado que la supervivencia cultural que había sido propia del sistema eclesial y en este sentido es posible notar la presencia de un conflicto velado detrás de la relación entre la Iglesia e indígena que revela un desapego de los propósitos de uno y otro:

“....un deseo por rechazar a la Iglesia, la antigua institución, como articulación mediadora con el mundo externo y como ayuda material y guía ideológica, a favor de una interacción directa con las burocracias estatales [...] Estos hombres jóvenes [migrantes y jornaleros] 
que han crecido desde la era de la hacienda, están ansiosos por competir directamente, como ciudadanos e iguales, con la gente blanca de los pueblos del Valle Interandino para obtener recursos gubernamentales" (Ibíd.: 118).

Las relaciones constituidas en las alianzas establecidas entre el MICC y la Iglesia, tomando en cuenta sus vinculaciones en torno a la educación intercultural como al desarrollo (FODERUMA), representan la acumulación de un "capital político" inicial que le servirá posteriormente al MICC para desarrollarse y desenvolverse en escenarios provinciales y nacionales. Las relaciones de fuerza establecidas entre el MICC y la Iglesia implican un "campo de juego" donde dos actores negocian influencias; tanto la Iglesia se acopla a las lógicas del movimiento indígena y tanto el mismo MICC se ve regido, en varias ocasiones, por los apoyos, muchas veces institucionales de parte de la Iglesia, o muchas veces por "agentes" de la misma estructura eclesiástica que tienen relación directa con el MICC. De tal forma, se constituye una relación donde lo que prima es un juego de contingencias y acuerdos que determinan los habitus de los agentes ${ }^{3}$.

Otro aspecto que debe ser tomado en cuenta dentro de los antecedentes del MICC es el de la comunicación rural también impulsada desde la Iglesia. A inicios de la década del 80 , se inicia la organización de un sistema de comunicación campesina indígena mediante la instalación de una cabina radiofónica en cada organización campesino-indígena de la provincia con el objetivo de motivar la producción de programas informativos y educativos para que sean retransmitidos por Radio Latacunga. Esta iniciativa contó con el apoyo de varias instituciones entre las cuales se cuentan CIESPAL y la Iglesia a través Radio Latacunga (Dávila, 1998: 151).

En este sentido, la radio ha constituido un proceso de ida y vuelta en Cotopaxi siendo un medio a través del cual la población indígena no sólo podía estar informada sino también comunicar sus problemas, necesidades y demandas. Así, la instalación de cabinas radiofónicas en algunas comunidades permitía realizar transmisiones en vivo donde los comuneros hablaban de sus problemas, de sus tradiciones y de su organización social y política en quichua. La radio había constituido desde su creación un medio a través del cual se catalizaba la organización social; la radio, en los primeros momentos de aparecimiento en la escena rural habría constituido una forma de acceder a la comunicación, una forma globalizada de relaciones nuevas, constituía la nueva forma de "estar juntos" 4.

3 La Teoría de la Acción constituye el eje de la presente investigación entendiendo ésta como una teoría que: "toma en consideración las potencialidades inscritas en el cuerpo de los agentes y en la estructura de las situaciones en las que éstos actúan o, con mayor exactitud, en su relación. Esta filosofía, que se condensa en un reducido número de conceptos fundamentales, habitus, campo, capital, y cuya piedra angular es la relación de doble sentido entre las estructuras objetivas (las de los campos sociales) y las estructuras incorporadas (las de los habitus)" (Bourdieu, 1997: 7-8).

4 A través de la radio se produce un fenómeno similar al de la globalización. En el caso que interesa, el mismo análisis puede ser trasladado a la escala local del funcionamiento de las radios con alcance en zonas rurales; Barbero ocupa el término incorporándolo al proceso de inclusión / exclusión al interior de los procesos culturales: "Ligado a sus dimensiones tecno-económicas, la globalización pone en marcha un proceso de interconexión a nivel mundial, que conecta todo lo que instrumentalmente vale empresas, instituciones, individuos- al mismo tiempo que desconecta todo lo que no vale para esa razón. Este proceso de inclusión/exclusión a escala planetaria está convirtiendo a la cultura en espacio estratégico de compresión de las tensiones que desgarran y recomponen el "estar juntos" (Barbero, 2002: 6). 


\section{Organismos de desarrollo: coautores de la organización política}

La Misión Andina, que operó en Cotopaxi anterior a los proyectos del Ministerio de Agricultura con sus Proyectos Integrales de Desarrollo Agropecuario (PIDA) y los programas de Desarrollo Rural Integral (DRI), constituyó, para la población indígena, el primer contacto con un organismo de ajuste estructural que pretendía incorporar al conglomerado rural dentro de "lo nacional", integrando al indio económicamente, culturalmente y políticamente ya que "rechazaba cualquier forma de autonomía en nombre de la unidad de la patria" a través del modelo de "desarrollo comunitario" que aprovechaba y reconvertía las "propias características internas de las comunidades como elementos potenciadores y dinámicos del cambio (estructural)" (Bretón, 2000: 18-19).

El antecedente de la Misión Andina en Cotopaxi refleja una serie de delineamientos del panorama social en el cual se movería la organización indígena. El "modelo de desarrollo comunitario" llevado a cabo por la Misión Andina, por un lado, no modificó el panorama de "condiciones de vida" de la gente del campo mejorando su economía y los servicios; y por otro lado no afectó estructuralmente a las organizaciones de base ya que las reforzó redireccionando sus propósitos. Cabe pensar que en esos primeros momentos de intervención en el área indígena, la comuna, primera instancia organizativa, tuvo una continuidad eficiente en lo que respecta a sus lógicas culturales internas, es decir, siguieron funcionando en su interior los lazos de reciprocidad y parentesco que la habían caracterizado manteniendo fija la base de la estructura (las comunidades) que más tarde tomará el MICC como punto inicial de organización.

Dentro de las planificaciones de reforma agraria a través de organismos estatales, el IERAC cumplió en los primeros años de la década del 70 una actitud modernizadora en la sierra ecuatoriana elevando los niveles de las fronteras agrícolas de las comunidades indígenas a través de la entrega de recursos específicos como la tractorización, los fertilizantes químicos y las semillas mejoradas. Así se esperaba intensificar la producción de productos para el consumo urbano interno; lo cual caracterizó, casi en su totalidad, la década del 70 (Santana, 1983: 72).

En este sentido, los programas de Reforma Agraria llevados a cabo directamente por el Estado a través de las acciones del IERAC y del PIDA en los años 70 diseñaron sus particularidades en torno a la marginación del medio campesino indígena cooptando sus potenciales productivos con el fin de rellenar los agujeros de oferta de productos que se habían establecido en los centros urbanos. De tal manera, los programas reformistas constituyeron un elemento político que debe ser considerado entendiendo la dimensión de su acción en el medio rural, en tanto ejercieron acciones orientadoras en la producción modificando estructuralmente las formas de vida al interior de las comunidades indígenas de base y propiciando un resquebrajamiento inicial de las tierras comunales a través del recorrido de la frontera agrícola en los páramos.

Posterior a la presencia de la Misión Andina, aparecen los Proyectos Integrales de Desarrollo Agropecuario (PIDA) del Ministerio de Agricultura que tuvieron una presencia corta en la zona ocupando los últimos años de la década del 70. Estos proyectos no pudieron acceder al medio campesino indígena debido a la carencia de experiencias exploratorias 
que hubieran permitido evaluar sus posteriores efectos, sin ni siquiera proveer de alguna experiencia piloto que modelara posibles intervenciones futuras; hasta que en 1979 la sustitución del gobierno terminó por desestructurar los logros operativos que, aunque poco significativos, caracterizaron al Ministerio de Agricultura, principal organismo patrocinador de este tipo de programas (Santana, 1983: 72).

Los PIDA, encuentran una inmediata continuación en la aparición de los proyectos de Desarrollo Rural Integral (DRI) que inician a partir de la década del 80 con 17 proyectos establecidas por el Plan Nacional de Desarrollo 1980-1984 (Ibíd.: 73) de los cuales solamente uno se aplica en una zona indígena de la provincia de Cotopaxi, específicamente en el cantón Salcedo. Además de este proyecto se implementó otro en el área de Tanicuchi, Toacazo y Pastocalle pero que no involucraba población indígena.

Los DRI incluyeron en sus acciones de asistencia una aproximación hacia los mismos principios llevados a cabo por los PIDA, incorporando una visión propia del último gobierno militar en temas de asistencia rural a través de la Ley de Fomento Agropecuario de 1979, que tenía como objetivo favorecer a las formas de producción capitalista apoyando su reconversión productiva y teniendo en cuenta, como factor importante para solucionar la presión demográfica, a la colonización. Además, los DRI incorporaron en sus dinámicas algunas formas de programas sociales laterales, infraestructura y tecnología incentivando policultivos de largo plazo, olvidando el autoconsumo campesino (Santana, 1983: 76).

Los proyectos de Desarrollo Rural Integral, constituyeron un fracaso más en el alcance del Estado hacia mejorar la calidad de vida de los campesinos. Al dirigir sus acciones hacia un campesinado con capacidades productivas viables, es decir, poseedores de tierra, los DRI se internaron en la sierra impulsando actividades de ganadería, comercialización, incorporación de tecnología; basando todos estos supuestos en la fuerza de la organización comunal del mundo andino e ignorando el problema real de la tierra (Martínez, 2002: 201).

El caso de Salcedo ilustra claramente la incapacidad de cambio rural que tuvieron los proyectos DRI y la visión de los campesinos sobre estos proyectos frente a sus condiciones de vida. Una visión generalizada de parte de los campesinos en la zona de Salcedo apela a la autogestión: "la solución de nuestros problemas no viene de afuera" y a la organización: "la solución está en el trabajo en grupo, regional, que nos ayudará a analizar nuestros problemas mejor y a buscar soluciones a corto y largo plazo". Y ante esta apelación por intenciones concretas es la UNOCAPAC (Organización de segundo grado que operó en la zona en aquella época), quien se dispuso a ser la forma de contacto inmediato con los proyectos DRI (Gangotena y Albán, 1982: 126-128).

Es importante señalar la acción llevada a cabo por el DRI Salcedo al momento de diseñar y programar un proyecto de agua en Cusubamba: el proyecto se llevó a cabo "sin los campesinos y, además, dejó fuera del proyecto a la gran mayoría de comunidades. La UNOCAPAC inicialmente solo oyó -con impotencia y admiración- que el agua no les llegaría" (Gangotena y Albán, 1982: 129).

Analizando esta relación asimétrica entre la organización de beneficiarios de los proyectos (UNOCAPAC) y la entidad ejecutora del mismo (DRI), se puede identificar una incapacidad gestionadora de parte de la OSG y una actitud hegemónica por parte del organismo estatal. Es importante señalar además que la opción de autogestión y 
organización sugerida más arriba dará gérmenes a la futura organización cantonal y provincial, en tanto algunas de las organizaciones fuertes del actual MICC provienen de Salcedo.

\section{Las instituciones privadas de desarrollo.}

A partir de la década del 80 trabajan, por otra parte, algunas ONGs sin vinculaciones estatales; su trabajo se centra básicamente en proyectos de desarrollo productivo. Entre estas organizaciones se encuentran el CAAP (Centra Andino de Acción Popular) que, además de los proyectos productivos, llevó adelante un proceso de documentación para la discusión teórica de los problemas rurales de Cotopaxi que ha contribuido para el debate agrario y rural en las ciencias sociales. Por otra parte SWISSAID y la CRUZ ROJA Suiza han manejado proyectos productivos en algunas áreas de la provincia. Una serie de instituciones empiezan la participación en este período de tiempo, unas más pronto que otras y con mayor y menor intensidad en el grado de intervención. Algunas de estas ONGs que han llevado a cabo procesos con el MICC o con Organizaciones filiales del MICC son: HEIFFER, IEE, ECOCIENCIA, DYA, APN, COMUNIDEC, PLAN INTERNACIONAL, FUNDEAL, entre otras.

El proceso político actual del MICC ha estado acompañado de varias de estas instituciones privadas de desarrollo, algunos de cuyos aspectos es importante recuperarlos. Para entender el proceso de vinculación de las ONGs con el movimiento indígena, el caso del Plan participativo de desarrollo de Cotopaxi (PPDPC) que lleva adelante el Consejo Provincial es ilustrativo en la medida en que las ONGs han tenido el papel fundamental en el equipo de apoyo externo conformado en torno al plan. Cabe resaltar que el caso del PPDPC es muy próximo al MICC ya que las políticas del Consejo Provincial mantienen una consulta directa y permanente con el MICC.

El PPDC, consiste en la instauración de un modelo de gestión administrativa para el desarrollo de la provincia donde se involucran tres conceptos principales: la transparencia, la democratización y la gestión para el desarrollo de Cotopaxi (Ospina, 2006: 34). Si vemos los términos ocupados en la definición de la tarea del plan advertimos un lenguaje propio de las nuevas prácticas de participación ciudadana y desarrollo local que están muy vinculadas a ONGs que apoyan los procesos. En el siguiente extracto de la sistematización de la Convención Ambiental ${ }^{5}$, se puede ver la participación activa de las ONGs en el proceso:

"Al interior del sector indígena se constituye un espacio de debate, acompañamiento y liderazgo político que se denomina "Equipo Externo" (conformado por ex dirigentes, líderes e intelectuales), las funciones de este colectivo son acompañar al Prefecto en la construcción de este nuevo proceso de gestión y además ser el puente entre la gestión pública y los objetivos de la organización" (Reyes, 2005: 8).

5 La Convención Ambiental es una iniciativa que parte de la Prefectura con el apoyo técnico y financiero de varias ONGs; esta iniciativa se constituye como un espacio de discusión y reflexión sobre la gestión de los recursos naturales en la provincia. 
La delineación de las "hojas de ruta”, por parte de las ONGs, que guían la acción de los espacios donde el MICC ha obtenido poder, muestra la importancia que tienen éstas en el desempeño del prefecto. La alianza del MICC a través de Umajinga y el Consejo Provincial responde a una alianza estratégica; la consolidación técnica de un programa de desarrollo local como un instrumento político para consolidar el poder es la piedra angular que mueve todos estos procesos de democratización y participación desde los gobiernos locales en una provincia históricamente parcializada por el manejo del poder entre quienes lo detentaban desde las esferas dominantes y entre quienes simplemente eran sectores subalternos de la política local.

Desde esta perspectiva cabe advertir que la consolidación de un frente aliado del MICC y de la organización indígena provincial en su totalidad por parte de las ONGs implica una forma de gobernar innovadora. Como lo señala Larrea $(2005,67)$ a partir de la aprobación de la propuesta para el financiamiento por parte del PRODEPINE del Plan participativo de desarrollo de Cotopaxi:

"Se da un primer logro significativo, pues el solo hecho de que ONGs con distintas identidades, dinámicas y prácticas institucionales conjuguen sus experticias, capacidades y recursos económicos frente a una propuesta conjunta, implica un cambio en las lógicas tradicionales del desarrollo, rebasando el ámbito de acción parcelado hacia dinámicas de gestión más integrales. Más aún si se considera que la propuesta de Cotopaxi constituye la primera experiencia de esta naturaleza en el país" (Larrea, 2005: 67).

\section{La protesta. Levantamientos, conquistas y pérdidas políticas.}

El levantamiento indígena de 1990 significó para el MICC, al igual que para gran parte de las organizaciones indígenas provinciales, una reestructuración del sentido de la lucha que venían adoptando décadas atrás; además de transformarse en brazo estructurante, de gran trascendencia, de la filial de la CONAIE en la sierra: la ECURUNARI. Lo que se sugiere es que el MICC reflejó, durante el levantamiento y buena parte de la década del 90, mucho de lo que había hecho y dicho la CONAIE y el Movimiento Indígena Nacional adoptando planteamientos paralelos pero en la escala local.

Por otra parte, este fenómeno puede ser leído entendiendo que la fuerza de la CONAIE en los primeros años de la década del 90 significaba la existencia de una vinculación directa con las organizaciones de tercer grado y éstas, a su vez, con las organizaciones de segundo y primer grado; consolidando una comunicación efectiva entre las dirigencias nacionales y las locales. Muchos de los líderes indígenas nacionales estuvieron muy vinculados a Cotopaxi en los preparativos previos al levantamiento de mayo del 90, incluso la $\mathrm{V}$ Asamblea de la CONAIE se realizó en Pujilí en febrero del mismo año, donde se planificó el levantamiento de mayo y se elaboró el planteamiento de 16 puntos que se incluye en el mandato por la vida (Guerrero y Ospina, 2003: 36).

En el levantamiento de 1990, junto con Chimborazo, Cotopaxi fue la provincia que mayor número de personas movilizadas alcanzó, además de ser la primera provincia que, a través de su organización local, el MICC, convocó a sus comunidades de base y bloquearon la carretera Panamericana interrumpiendo el transporte arterial del país como una forma de exigencia de las demandas planteadas a nivel nacional por el movimiento indígena. 
Este levantamiento fue planificado con anterioridad ante el llamado de la ECUARUNARI y la CONAIE; las organizaciones que participaron activamente en la planificación fueron: UNOCIZ, UNOCAM, UNORIG y UNOCANC; y quien estuvo a la cabeza fue Leonidas Iza, de la UNOCANC y Miguel Proaño de la UNOCAM; además se contó con la colaboración de algunos asesores mestizos que venían acompañando el proceso del MICC desde años atrás ${ }^{6}$.

En torno a la planificación del levantamiento en Cotopaxi es importante advertir el trabajo que significó la comunicación a las bases; algunos miembros del MICC recorrían por las comunidades comunicando la necesidad de salir a protestar y escuchando los planteamientos de las bases, esto significaba un proceso de largas conversaciones con los comuneros de las zonas alejadas que no tenían información de lo que sucedía y de las resoluciones que se tomaba en las reuniones (Entrevista J. T.) ${ }^{7}$.

Por otro lado, es importante señalar que la radio jugó un papel muy importante en el levantamiento, ya que viabilizó la difusión de las convocatorias a las bases para salir a las reuniones previas a la movilización. A través de la radio, un medio identificado por la gran mayoría de pobladores rurales de las áreas periféricas de la provincia cuyas inquietudes y necesidades habían sido "sacadas al aire" por medio de la emisora, se empezó a gestar una opinión pública (indígena) en torno al levantamiento; traduciéndose esto en la gran acogida que tuvieron las convocatorias realizadas a través de la radio.

\section{La lucha por el respeto y el reconocimiento}

En Cotopaxi el levantamiento de 1990 significó un reflejo de los descontentos anunciados por la CONAIE y por la Coordinadora de Conflictos Agrarios. El tema del respeto y trato diferente al indígena por parte de la sociedad blanca mestiza, básicamente en las cabeceras cantonales y la capital de la provincia, había significado una posición semejante a lo demandado por la CONAIE en términos generales para el movimiento indígena nacional; y por otra parte, al ser Cotopaxi una provincia que concentraba un número considerable de conflictos agrarios, el tema de la tierra y el territorio había significado un aspecto relevante al momento de unificar los descontentos de las bases que clamaban por una tierra propia para cultivar y un equilibrio razonable en el precio de la producción obtenida para regenerar, por lo menos en algo, la pauperizada vida de los campesinos en las áreas rurales. Es ilustrativo para comprender la dimensión del problema una declaración de una campesina de Pastocalle donde dice: "Nosotros tenemos que vender unos 10 o 20 quintales de papas para comprar un sólo quintal de abono, señor. Los fertilizantes, ¡semejante caro! Nosotros, nuestros hijos, estamos muriendo de hambre, por eso hemos salido" (Rosero, 1990: 10).

6 Testimonio de Baltazar Umajinga, en Tibán et al., 2003: 83.

7 Miembro de la OSG COJACAP de Pujilí, ex Presidente de la misma organización y ex Secretario del MICC; participa activamente en las actividades del MICC y de su OSG; Poaló, en el contexto del 8vo Congreso del MICC, 8 de Julio de 2005. 
Las demandas en torno al respeto hacia la población indígena fueron planteadas en el contexto de la participación en el levantamiento. Varios de los testimonios referidos en torno a la movilización mencionan las motivaciones que llevaron a los indígenas de Cotopaxi a movilizarse. En relación a la protesta llevada a cabo en el contexto del levantamiento en la plaza de El Salto en Latacunga frente a la gobernadora, el presidente del MICC de aquel entonces, Baltazar Umajinga, dice:

"El planteamiento de todos los que hablaron frente a las autoridades, era que se respete a la población indígena, que no nos maltraten como a los ladrones, que nos reciban en las oficinas sin sacar sombrero, etc.” (Tibán, 2003: 83).

Por otra parte, los recuerdos de los tiempos anteriores al levantamiento y anteriores a las reivindicaciones logradas por el Movimiento esbozan de manera clara el tipo de trato que se producía ante los indígenas de parte de la población urbana: "Antes era terrible, antes el indígena era, hablando vulgarmente, un bicho así que no podía estar pegado al mestizo" $(\text { Entrevista, B. P. })^{8}$. Durante los primeros momentos del levantamiento, la posición de la sociedad urbana en las cabeceras cantonales y la capital de la provincia seguía siendo de incredulidad, de negación hacia la posibilidad de protesta del Movimiento Indígena. Un testimonio de José Toapanta, quien participó activamente en el levantamiento ilustra claramente como percibían los urbanos a los indígenas, desde su punto de vista: "La gente de la ciudad al igual que los militares nos veían como algo folklórico, nos vieron como un juego o una burla, no sabían qué es lo que iba a pasar, no sabían que los indios iban a mover el mundo con ese levantamiento" (Tibán, 2003: 73-74).

El proceso histórico del pueblo indígena en Cotopaxi refleja una relación de dominación permanente tanto desde el Estado, a través de sus estructuras extendidas local y nacionalmente por medio de instituciones públicas, como de las clases privadas dominantes a través de las clases hacendatarias, los intermediarios comerciantes, los transportistas, etc. Esta dinámica de dominación implica una constitución de un habitus particular en torno a los agentes movilizadores, es decir, los individuos que participaron en el levantamiento. Es esa conjunción de habitus compuestos en torno a una determinada constitución de un campo de juego, es decir, de un escenario (el de la dominación) lo que importa al momento de entender el por qué de los reclamos en el levantamiento del 90, y el por qué de la efectividad en la acción movilizadota. Coyunturalmente la acción movilizadora del levantamiento de 1990, implicó una aceptación inmediata y positiva de parte de las organizaciones de segundo grado, de las mismas comunidades y de los mismos comuneros identificados con una causa común: la de la exigencia de respeto y reconocimiento.

La demanda por respeto y reconocimiento manifestada por los líderes indígenas locales $^{9}$ pertenecientes al MICC, que confluyó en una gran aceptación por las comunidades

8 B. P. forma parte del grupo de mujeres de Cusubamba y ha sido una líder histórica del MICC; Latacunga, 22 de Septiembre de 2005.

9 Para una exposición más amplia de los testimonios sobre las motivaciones para llevar adelante la movilización ver Tibán, 2003 . 
de segundo grado y las bases, implicaba el planteamiento más general y amplio de declarar al Estado ecuatoriano como "Pluricultural", modificando el primer artículo de la constitución política de la república (Almeida, 1993: 167-168). Este planteamiento de la interculturalidad, tiene que ser percibido desde una perspectiva que reconozca la capacidad de cambio en la estructura estatal que puede ocurrir a partir de la acción de un movimiento social eficaz. En este sentido, es interesante tomar el planteamiento de Touraine al identificar los movimientos sociales como generadores de acciones colectivas mediante las cuales se pueda modificar las relaciones de dominación sobre los recursos culturales, es decir la modificación (la corrección) de una norma o un valor de la sociedad (Touraine, 1984: 94).

Sin embargo la efectiva acción movilizadora alcanzada por el MICC en el plano del levantamiento de 1990 implicó una apelación al Estado, una apelación a la inclusión de parte del Estado de unas demandas particulares (las del respeto al indígena a través del planteamiento de la plurinacionalidad), pero que simplemente se dirigió a corregir un elemento "enfermo" del Estado pero no de la sociedad. En este sentido, este primer momento de aparición del MICC como actor definido implicó el reconocimiento, de parte de la sociedad local, simplemente de la existencia de un movimiento consolidado pero que no lograría cambios significativos en el devenir cotidiano de las normas y las reglas sociales. Un planteamiento similar mantiene Ospina al afirmar que:

"El cambio en las estructuras elementales del racismo cotidiano depende de multitud de transformaciones moleculares en actitudes de cientos, miles, millones de agentes individuales y grupos dispersos. La ley de la costumbre no se cambia por la ley del Estado" (Ospina, 2006: 34).

La fuerza que tuvo el levantamiento de 1990 en Cotopaxi adquirió un significado estructural en la organización provincial. A partir del levantamiento se reconoció al MICC, no sólo por parte de la sociedad urbana blanco-mestiza de Cotopaxi, como organización política indígena, sino también para los propios indígenas el levantamiento constituyó un hito clave en el desarrollo político. A partir del levantamiento las cosas empezaron a caminar mejor al interior de la organización; desde ahí se entendió que el movimiento podía tener fuerza y que tenía la capacidad de generar propuestas y convocar a las bases.

El ejercicio de lo político por parte del grupo, en este caso del MICC como estructura a partir del levantamiento del 90, puede ser interpretado desde una visión de la antropología política procesualista, a través de la cual este ejercicio depende de la implementación de objetivos públicos al interior de un grupo determinado de la sociedad. Estos objetivos públicos, en el caso del MICC, estarían traducidos en la lucha por el derecho a ser reconocidos y ser respetados. Aquellos objetivos públicos "para” los miembros del MICC implican "el establecimiento de una nueva relación con otro grupo o grupos" (Varela, 2005: 100); que vendría a ser la sociedad blanco-mestiza, que no otorga el reconocimiento y el respeto al indígena y que son el contenido central de las demandas para la adquisición de un determinado poder que irá aumentando hasta años posteriores en que se participa electoralmente y se accede a cargos importantes como la prefectura o la alcaldía. 


\section{La vieja demanda por la tierra}

Por otra parte, como habíamos señalado más arriba, se distinguía otro elemento que había funcionado como causa para que se produzca la movilización: los conflictos por la tierra. El tema de la tierra en Cotopaxi significó un proceso de largos años de confrontaciones y demandas; desde el tiempo de la reforma agraria hasta los años 80 , como se vio en el capítulo anterior, las luchas agrarias habían sido un elemento que motivaba la organización.

La presencia del tema de la tierra como una necesidad urgente de reivindicación aparece frecuentemente en el discurso de los indígenas de Cotopaxi; Baltazar Umajinga, presidente del MICC posesionado meses después del levantamiento se refiere a la cuestión de la tierra en los siguientes términos:

"En la lucha por la tierra el objetivo más grande fue recuperar las tierras que los hacendados nos robaron, porque sabíamos que antiguamente los dueños fueron nuestros abuelos y pasaba de generación en generación” (Tibán, 2003: 82).

En este contexto, cabe referirse al problema de la tierra como un elemento claramente movilizador y catalizador del levantamiento del 90 ya que es considerado, en el discurso interior de los actores, un tema que ha sido postergado desde la época de la Colonia y la República.

Dentro del contexto del primer año de gobierno de la Izquierda Democrática, las resoluciones de inafectabilidad presentaban cuadros altos que fueron, de alguna manera, un detonante para la movilización del 90. El caso de Cotopaxi tiene ciertas particularidades al ser conjuntamente con Pichincha, Imbabura y Chimborazo una de las provincias que mayor número de hectáreas incluyó en las resoluciones de inafectabilidad.

\begin{tabular}{|l|c|c|c|c|c|c|c|}
\hline \multicolumn{7}{|c|}{ Cuadro 1. Resoluciones de inafectabilidad en la Sierra } \\
\hline Provincia & número & has & Nd & $\mathbf{0 - 1 0}$ & $\mathbf{1 0 - 1 0 0}$ & $\mathbf{1 0 0 - 5 0 0}$ & Más de 500 \\
\hline Carchi & 12 & 871,35 & 3 & 0 & 4 & 5 & - \\
\hline Imbabura & 15 & 1425,12 & 6 & 0 & 4 & 4 & 1 \\
\hline Pichincha & 40 & 5117,74 & 7 & 8 & 15 & 7 & 3 \\
\hline Cotopaxi & $\mathbf{6}$ & $\mathbf{1 6 0 4 , 4 8}$ & $\mathbf{0}$ & $\mathbf{1}$ & $\mathbf{3}$ & $\mathbf{1}$ & $\mathbf{1}$ \\
\hline Chimborazo & 12 & 1302,77 & 1 & 3 & 6 & 1 & 1 \\
\hline Bolívar & 2 & 307,07 & 0 & 1 & 0 & 1 & 0 \\
\hline Cañar & 4 & 193,12 & 0 & 1 & 2 & 1 & 0 \\
\hline Azuay & 9 & 3856 & 1 & 0 & 4 & 0 & 4 \\
\hline Loja & 7 & 83 & 6 & 0 & 1 & 0 & 0 \\
\hline Sierra & $\mathbf{1 0 7}$ & $\mathbf{1 4 7 6 0 , 6 5}$ & $\mathbf{2 4}$ & $\mathbf{1 4}$ & $\mathbf{3 9}$ & $\mathbf{2 0}$ & $\mathbf{1 0}$ \\
\hline
\end{tabular}

Elaboración: José Egas / Fuente: IERAC; (Rosero, 1990: 41). 
Las resoluciones de inafectabilidad en Cotopaxi entre agosto de 1988, año en que asume la presidencia Rodrigo Borja, y julio de 1990, momento en que se produce el levantamiento indígena la ubican como la segunda provincia que mayor número de hectáreas tratadas por las resoluciones de inafectabilidad tiene después de Pichincha. El número de hectáreas con inafectabilidad de tierras es de 1.604 .48 en contraste con el número de resoluciones que es mucho menor al resto de provincias, en Cotopaxi las resoluciones de inafectabilidad son sólo 6 mientras que, por ejemplo en Chimborazo con un número igualmente alto de hectáreas inafectadas (1.302.77), el número de resoluciones es de 12. Esto demuestra que en Cotopaxi el problema de las tierras inafectadas está claramente concentrado en unas pocas propiedades grandes ( 2 de entre 100 y más de 500 hectáreas) y algunas medianas (3 de 10 a 100 hectáreas). Por otra parte cabe señalar que la mayor cantidad de conflictos de tierra que se suscitaron en Cotopaxi estaban concentrados en los cantones que habían tenido mayores intervenciones por la Reforma Agraria; en ese contexto Pujilí fue el cantón que aglutinó la mayor cantidad de conflictos recientes (Ibarra y Ospina, 1994: 97).

A través de estos datos se puede inferir que el problema de la tierra en Cotopaxi se basó en conflictos frente a grandes propietarios debido a la concentración de extensión en pocas propiedades y la magnitud de hectáreas inafectadas. La defensa del latifundio, aquellos grandes remanentes de tierra, en el transcurso de los dos primeros años del gobierno de Borja parece haber sido real en la medida en tanto hubo, además, una paralización de los verdaderos procesos de Reforma Agraria, ya que los conflictos por tierra identificados por Rosero (1990: 44) demuestran su predominio, al menos en Cotopaxi, en propiedades con extensiones grandes: de 70 conflictos en total, 24 se encuentran en propiedades entre las 10 y 100 hectáreas, 17 en propiedades entre las 100 y 500 hectáreas y 9 en propiedades de más de 500 hectáreas. Entre los conflictos estudiados por Rosero en Cotopaxi sobresalen las zonas de Pujilí, Saquisilí y Salcedo.

Sin embargo, es importante también el número significativo de conflictos con pequeñas propiedades de menos de 100 hectáreas. Este cambio es interesante mirarlo si se lo compara con los litigios producidos en los años 60 y 70, donde los conflictos se establecían entre campesinos y grandes propiedades de 500 o más hectáreas (hasta de 15000 hectáreas como en los casos de la hacienda La Lorena y algunas en la zona de Angamarca). En este sentido se puede decir que los conflictos redujeron el tamaño de las superficies reclamadas que consistían en conflictos más puntuales por pequeños predios en posesión o por páramos indivisos; además que estos conflictos se insertaban en el reclamo por tierras de mala calidad siendo las tierras demandadas páramos inadecuados para la agricultura, zonas laderosas o con grandes pendientes hacia los declives de la cordillera occidental (Ibarra y Ospina, 1994: 98).

Además de la limitación de acceso a la tierra ocupada, en su mayor parte de extensión, por las grandes propiedades; el problema de la tierra, manifestado en algunos conflictos agrarios específicos, asistía a una confrontación directa con la clase hacendataria. Los hacendados seguían oponiéndose a cualquier "progreso" del indígena; C. E. ${ }^{10}$, presidente del MICC en el anterior período al actual, recuerda que en los tiempos anteriores al

10 C. E. al momento de la entrevista era presidente del MICC; pertenece a Poaló. Latacunga, 12 de Agosto de 2005. 
levantamiento de 1990 "había una expectativa por la lucha de la Tierra, se quería acabar con la hacienda porque era un obstáculo para la profesionalización que era mal vista por el sector hacendado" (Entrevista C. E.).

La información detallada en las líneas anteriores demuestra la importancia de los conflictos en torno a la tierra que se produjeron en Cotopaxi en los años previos al levantamiento de 1990. Un punto fundamental y que debe ser considerado, es los ofrecimientos de la campaña electoral de un gobierno distinto con aproximaciones más reales al mejoramiento de las condiciones de vida de los campesinos; ofrecimientos que no fueron tomados en consideración sino hasta la negociación que siguió al levantamiento en la cual se accedió a ciertas demandas expuestas como condiciones por parte de la CONAIE ${ }^{11}$.

\section{La participación. Los juegos electorales.}

La provincia de Cotopaxi, ha constituido uno de los espacios donde el movimiento indígena se ha manifestado como una expresión de triunfo en lo que se refiere a la participación electoral. El Movimiento Pachakutik ha funcionado con éxito en las áreas rurales de la provincia, obteniendo triunfos electorales importantes que han permitido una ubicación favorable frente a las demandas del sistema democrático ecuatoriano. Manifestaciones de ello han sido los múltiples logros alcanzados desde 1998, en el acceso al control de ciertas instituciones estatales y la ocupación de un porcentaje significativo de cargos públicos en los gobiernos seccionales.

Los indicadores electorales que exponen el éxito de Pachakutik en Cotopaxi, sobre todo en las áreas con mayor población indígena, sugieren pensar en una serie de dinámicas que se producen al interior de este fenómeno. La elección de los candidatos que representarán al Movimiento Indígena a través de su brazo político Pachakutik, implica una relación dinámica entre las bases y los liderazgos. Lo que se podría pensar, es que se da un distanciamiento pronunciado entre los dos extremos del movimiento a partir de la participación en las elecciones ya que se desarrolla un juego partidista al cual el movimiento, como estructura, no estaba "habituado"12. En este sentido, se constituye la necesidad de establecer perfiles sólidos que sepan manejar el juego político de los partidos para poder competir al mismo nivel con las demás fuerzas políticas representadas en partidos de larga trascendencia administrados, en su gran mayoría, como corporaciones caudillistas con una acumulación de capital económico y político funcional.

Las dinámicas al interior de los gobiernos seccionales manejados por indígenas, generalmente de Pachakutik, en las provincias ecuatorianas han consistido en llevar a cabo administraciones apegadas al desarrollo local impulsando prácticas de participación ciudadana a través de asambleas cantonales y otras formas institucionales incluyentes de

11 Para un seguimiento más detallado del cumplimiento o incumplimiento de las demandas de la CONAIE por parte del gobierno (Ortiz, 1992: 174-177).

12 Habituado en el sentido de habitus como "sistemas de disposiciones duraderas y transferibles, estructuras estructuradas predispuestas para funcionar como estructuras estructurantes” (Bourdieu, 1991: 93). 
la sociedad civil a través del concepto de la ciudadanía. Muchas de estas prácticas llevadas a cabo por los indígenas en los gobiernos locales han sido impulsadas por ONGs que han "acompañado" los procesos políticos del movimiento indígena. En este sentido la participación electoral como un primer momento de acción implicó un triunfo político y acumulación de capital; sin embargo, al momento de consumarse el triunfo, el movimiento indígena tiende a adoptar tendencias convencionales de administración de los gobiernos locales; convencionales en la medida en que son formas de acción muy similares a las llevadas a cabo por gobiernos seccionales de otros partidos tradicionales.

Lo que sugerimos es que esas prácticas han formado parte de construcciones mediadas por agentes externos de desarrollo, básicamente traducidos en las ONGs privadas cuya presencia en la década de los ochenta empezaría a ser reiterativa en la escena rural ante la privatización y des-estatización del desarrollo. El punto que se ha sugerido, implica el planteamiento de si las ONGs han promovido o no acciones que hayan tenido efectos positivos o negativos en el mejoramiento de la calidad de vida de la población rural. Esa posibilidad queda planteada en el contexto de la existencia de una importante cantidad de bibliografía sobre el tema que puede ser resumido en dos posturas: quienes creen que la acción de las ONGs no ha sido funcional al sistema neoliberal de ajuste, sino que ha contribuido a la conformación de un desarrollo alternativo y quienes creen que las acciones de las ONGs en el campo no han tenido una influencia positiva en el desarrollo económico de la población ${ }^{13}$.

Por otro lado, las consecuencias del triunfo electoral a nivel local sugieren pensar en la relación entre las autoridades indígenas elegidas para ocupar cargos y las bases del movimiento. Cabe manifestar la inquietud de si se producen aún desde las nuevas autoridades indígenas, tal vez obedeciendo a la reproducción de prácticas que se habían enquistado en las instituciones durante la administración de los sectores burocráticos tradicionales de los partidos que habían alcanzado puestos públicos en gobiernos locales, prácticas clientelares que pudieran ser causas de la erosión entre dirigencia y bases en tanto significan procesos políticos ajenos a los sistemas tradicionales andinos.

\section{La participación electoral. Continuidad del liderazgo o verticalización del poder.}

La historia de la organización nacional y que encuentra una expresión consecuente con lo que sucede en Cotopaxi saca a la vista el tema del liderazgo. El liderazgo indígena ha constituido uno de los elementos históricos de la organización indígena desde ciertos puntos de vista. En primer lugar la organización comunitaria andina cuyo lema ha sido el "mandar obedeciendo", ha significado pensar siempre en una decisión colectiva privilegiada en detrimento de la acción individual deliberada. Esa explicación está claramente manifestada en el modo de vida comunal que llevan las comunidades andinas y que se ha reproducido, al menos en discurso, al Movimiento Indígena Nacional. Desde esta perspectiva el planteamiento de liderazgos implica el surgimiento de un nuevo elemento dentro del modelo de organización.

13 El debate entre estas dos posturas se ilustra bien, además de sugerir algunos otros datos bibliográficos sobre el tema, en la discusión entre Ospina (2002) y Bretón (2002). 
Una retrospectiva histórica del liderazgo obliga a mirar el tema de las comunas, en cuyo interior las lógicas de reciprocidad y parentesco manifiestas en instituciones como la minga, entre muchas otras, constituyen leyes culturales que regulan la sociedad. Como habíamos visto al tratar el tema de la comunidad, la instancia política de la comuna como figura jurídica implicó un primer acercamiento al liderazgo en tanto nombraba como miembros del cabildo a un presidente, vicepresidente, tesorero y síndico. Estos nombramientos significaron la ocupación de espacios tradicionales, propios del sistema de Estado moderno en los que se privilegia la existencia de líderes y clases dirigentes.

Con la evolución de las estructuras comunales y el surgimiento de las federaciones a más amplios niveles, el papel del "dirigente" tomó posición en las esferas del movimiento indígena. Por otra parte, muchas de las lógicas del desarrollo que han estado presentes en Cotopaxi durante los últimos treinta años a través de las distintas agencias, primero estatales y luego privadas, han iniciado procesos de capacitación donde fomentan la formación de líderes para que conduzcan a los grupos que están involucrados con los proyectos que se llevan a cabo. Después de todo, el elemento más notable de la naturaleza de las prácticas de desarrollo es justamente su acción intervencionista con el objeto de modificar algo en la sociedad afectada.

El caso del liderazgo indígena, debe ser analizado en la perspectiva de una transformación en la organización política estructural del mismo movimiento. La formación de líderes políticos fuertes en cada organización, implica la existencia de una racionalidad política sincrética donde intervienen elementos del sistema de representación moderno y el sistema de representación tradicional de la cultura comunal andina. En este contexto, el interés en la formación de líderes implica y debe ser pensado como una dinámica estratégica de posicionamiento dentro del sistema de representación vigente en el Estado moderno. Esta tendencia de acumular el poder en los líderes responde, por otra parte, a la necesidad de adaptación del movimiento indígena a las demandas del sistema político democrático que rige en el Ecuador. En esta medida, a partir de las elecciones, lo que se produce es un distanciamiento marcado entre las élites y las bases en tanto las élites están respondiendo a su adaptabilidad al sistema político que, sin embargo, merma, por lo menos en un nivel reducido, los elementos de un discurso caracterizado por la permanente manifestación de que en las estructuras del movimiento aún se actúa bajo el slogan de "mandar obedeciendo".

En este sentido es ilustrativa la afirmación de Santana:

"Se ha producido la constitución de una clase dirigente en el mismo seno de la sociedad indígena, formada de intelectuales, de cuadros políticos, de profesionales y de técnicos que asumen el control de las múltiples organizaciones que ha producido la dinámica étnica a partir de los años 70" (Santana, 2004: 70).

La capacitación es un elemento indispensable para el acceso a la participación como candidatos en las contiendas electorales, lo que demuestra que la estrategia política de ubicación en el campo político implica la "habituación", la acumulación de capital social (por medio de la educación, las capacitaciones, los cursos, etc.) y capital político (por medio del ejercicio político). Desde esta visión, una dirigente indígena de Cotopaxi afirmaba como es necesaria la experiencia para poder "jugar el juego" y aplicar las estrategias necesarias: 
"Yo digo, también es bueno que vaya saliendo gente, que se vaya creando a los nuevos dirigentes, pero también hay que tomar la experiencia de la gente que ya ha venido participando, para que vayan aportando sus ideas, entonces eso es lo que estamos ahora tratando de trabajar más" (Entrevista B. P.).

\section{Distribución y elección de candidatos a dignidades y dirigencia.}

El llegar a ser candidato representante de Pachakutik u ocupar puestos en la dirigencia del movimiento indígena local (MICC), implica tener una trayectoria de liderazgo que se construye desde tempranas edades a través del involucramiento en actividades políticas y de desarrollo. La gran mayoría de los líderes indígenas en el contexto nacional y local registran procesos de participación en la organización desde su juventud. El testimonio de C. G. es interesante desde este punto de vista porque recoge su trayectoria como una dirigente importante del MICC y de Pachakutik:

"Desde que yo tenía 22 años vine participando con mi comunidad, desde ahí he ido prestando servicios en la escuela cuando mis hijos eran alumnos, de ahí pasé a la junta parroquial que fui vocal, después fui dirigente del Comité de Aguas, después desde el año 1998 vine participando en la organización de MICC y luego pasé a ser dirigente provincial, y he sido dirigente de varias áreas, actualmente soy dirigente de género del MICC" (Entrevista C. G.) ${ }^{14}$.

Cabe en este punto del análisis la perspectiva de la acumulación de capital que se requiere para llegar a ser candidato o dirigente; la experiencia importa al momento de tomar las decisiones; cuando se decide sobre la participación de algún candidato es importante que sea conocido y que haya trabajado conjuntamente con el movimiento para demostrar que realmente ha acumulado cierta cantidad de experiencia para poder desempeñar bien las exigencias de la dirigencia. En ese sentido, el ser dirigente o candidato implica una acumulación de habitus, de "sentido del juego" que permite saber las tácticas y estrategias que se deben acatar en momentos de toma de decisiones.

Un planteamiento tentativo propone que, por un lado, las dinámicas del desarrollo mediante sus lógicas intervencionistas a través de las capacitaciones para formación de líderes y, por otro lado, las condiciones de desempleo y subempleo que se producen en el campo, indicados en expresiones tales como la migración a las grandes ciudades, incentivada básicamente por la pauperización del sector campesino agrícola, han conducido a una dependencia de puestos de trabajo remunerado, primero, en agencias de desarrollo como promotores o técnicos locales (esos son los términos que se utilizan) en sus comunidades o zonas. Segundo, a partir de la entrada en las competencias electorales y el posterior triunfo y acceso a los puestos estatales como la prefectura y ciertas alcaldías, se ha permitido el reordenamiento de algunos puestos burocráticos que permiten el acceso a un número considerable de pobladores rurales próximos al MICC.

14 C.G. dirigente del MICC, y precandidata para ser dirigente provincial de Pachakutik, pertenece a la parroquia Chugchilán del cantón Sigchos. Ha dirigido capacitaciones a mujeres y ha estado muy vinculada con el proceso del MICC. Latacunga 28 de Agosto de 2005. 
Hasta la actualidad muchos de los proyectos, además de intervenir con su acción desarrollista, han abierto la posibilidad de trabajar en proyectos. El caso del Proyecto de Desarrollo de Cotopaxi, PRODECO, un proyecto de desarrollo de gran envergadura bajo el cual se estableció un convenio de financiamiento y asesoría entre la Unión Europea y el CODENPE, buscó sus beneficiarios directamente en el área de influjo sin establecer relaciones cercanas con ninguna OSG ni la dirigencia del MICC (Ospina, 2006: 79). Esto puede haber tenido un efecto en la promoción de puestos de trabajo a nivel de base familiar ( $\sin$ contar con las organizaciones definidas como la comuna o las OSGs), ya que, el proyecto implicaba la necesidad de gran cantidad de personal para su operación; el director nacional del proyecto, proviene de la dirigencia fuerte de Cotopaxi y es un ejemplo de la ocupación de cargos no sólo a nivel público sino también de ocupación y uso de empleo del desarrollo privado.

Para el análisis de la selección de los candidatos idóneos para participar tanto de la dirigencia provincial del MICC como en las elecciones de dignidades públicas estatales, es importante tener en cuenta los puntos antes mencionados en la medida en que sugieren que lo que se produce es un relativo apaciguamiento del subempleo a través de los proyectos de desarrollo, las organizaciones de segundo grado (OSGs), otras formas organizativas de base (comuna, juntas de agua, comités barriales) al captar proyectos de desarrollo, juntas parroquiales y dignidades provinciales (prefectura, alcaldías, concejerías y concejalías), que promueven el empleo de los pobladores locales y beneficiarios. Este planteamiento invita a pensar que si el empleo se concretiza efectivamente en estos espacios éste no es abundante y no alcanza "para todos". Esto sugiere que la práctica que aparece como legítima en esta dinámica es la de la circulación de los cargos, lo cual se manifiesta en varios testimonios de manera positiva o negativa, es decir, hay quienes cumplen con la reciprocidad en los cargos y quienes "acaparan" y no dejan entrar a nuevas personas (Entrevistas a M. A.; C. G., J. T., N. I., A. C. ${ }^{15}$ ).

El testimonio de A. C. con respecto a las candidaturas nos incentiva a pensar en nuevas perspectivas acerca de la circulación de puestos:

"Cuando yo fui electo para candidato para concejal de Saquisilí a mí me apoyaron como en 22 comunidades y yo gané; pero de algunas comunidades dijeron que por qué tenía que ser de la misma zona del alcalde, entonces yo desistí de mi candidatura para que la gente de otra comunidad entre. Es mejor que otra gente entre y yo sigo participando aparte. Hay otros en cambio que representan a una comunidad o dos comunidades y representan como candidatos, entonces, son pocas las personas que tiene ese respaldo, así, masivo porque todo depende de cómo trabajó cuando fue dirigente, cuando fue electo en una organización" (Entrevista A.C.).

15 M.A. Líder de la comunidad de Cochapamba y ha participado en el proceso organizativo del MICC, ha ocupado varios cargos, entre los cuales la presidencia del MICC y la vicepresidencia. 25 de Agosto de 2005.

A.C. rector del colegio Jatarishun y pertenece a la organización Jatarishun de Saquisilí; ha sido dirigente del MICC y candidato a concejal por el cantón Saquisilí; Saquisilí, 15 de Agosto de 2005

N.I. dirigente de la UNOCANC y dirigente del MICC en varios cargos y varios periodos. Poaló, en el contexto del 8vo Congreso del MICC, 8 de Julio de 2005. 
La manifestación de A. C., plantea la necesidad de circular los cargos y las opciones electorales y de dirigencia que muchas veces pueden obedecer a la cuestión del empleo antes mencionada o también a una estructuración de una plataforma política consistente para la participación electoral en diversas zonas; lo que se trata es de ir ganando terreno político y representatividad en diversas localidades de la provincia para que el MICC tenga un efecto político cohesionador al interior de cada cantón y de cada parroquia.

Este principio de circulación de los puestos, obedece a un principio más amplio de la cosmovisión andina de la reciprocidad: la solidaridad en "ceder los puestos a otros para que ellos también participen”, se ha extendido más allá de los perímetros culturales de la comunidad llegando a influir en las decisiones de cada organización y del MICC como instancia provincial. El segundo principio establecido en el estatuto y reglamento del MICC en el Art. 4, plantea la solidaridad y la reciprocidad como guías conductoras de las acciones del movimiento (MICC, 2002: 12); en esa medida el elemento cultural se instituye dentro de una figura que es representación plena de las acciones de la organización. Así un testimonio de B. C. ${ }^{16}$ dice:

"A veces algunos compañeros piensan de seguir en los puestos, otros dicen, ahora me toca a mí, ha habido ese tipo de problema, sin embargo eso hemos ido fortaleciendo. Todos tenemos derecho de ser alcaldes o concejales pero no al mismo tiempo que es lo que quieren algunos compañeros" (B.C.).

De igual manera sucede con Pachakutik Provincial que actúa muy próximo al MICC casi sin distinguir una diferenciación, las normas de elección de candidaturas que, por ejemplo, Lourdes Tibán, importante líder indígena de Cotopaxi y actual directora del CODENPE, señala que en el proceso de pos-candidatura, los candidatos tienen que cumplir ciertos requisitos entre los cuales uno llama la atención: "Dar alternabilidad en un determinado periodo a su alterno", requisito que se incorporó en las elecciones de 1998, cuando se iba a elegir diputados, concejeros y concejales. La alternabilidad se firmó con todas las autoridades con el fin de que el alterno no sea una forma de un simple cumplimiento del requisito electoral sino más bien como una forma de promover la participación igualitaria tanto del principal como del alterno (Tibán, 2004: 117).

Este acuerdo firmado entre las autoridades demuestra que la circulación de cargos públicos tiene que ir más allá de los perfiles particulares; en este caso la circulación se da entre dos figuras, el principal y el alterno. Sin embargo, este tipo de acuerdos como señala Tibán no fueron acatados por todas las autoridades que firmaron el compromiso aduciendo que están a órdenes de la constitución y la ley del Estado, lo cual lo ve como un problema del tema planteado con respecto a las opciones de empleo que significa la participación en este tipo de dignidades:

“...en los indígenas empieza a presentarse la confusión de lo que implica una participación política alternativa y lo que significa el ingreso económico, transformado a la participación política en una forma de empleo y de supervivencia familiar" (Tibán, 2004: 118).

16 B.C. fue presidente del MICC, sin embargo hasta ahora es apegado dirigente del movimiento; es miembro de la organización JATARISHUN de Saquisilí de la cual también fue presidente. Saquisilí, sede de JATARISHUN, 20 de Agosto 2005. 
La cuestión de la reelección de dignidades por dos o más períodos consecutivos tiene puntos en contra que, de igual forma que las dinámicas anteriores, bloquea esta circulación de los puestos planteando el rechazo al liderazgo tradicional del corporativismo partidario donde quienes aparecen a la cabeza de la organización son ciertos caudillos perpetuos que extienden su poder en espacio y tiempo. B. C., dirigente de larga trascendencia, plantea que:

“...la reelección perjudica bastante al movimiento, porque hay algunos resultados favorables y otros en contra porque si se habla de un movimiento alternativo tiene que darse paso a otros compañeros para que ganen espacio, pero a veces algunos compañeros no entienden eso y quieren quedarse en el puesto" (Entrevista B. C.).

Algunas de las características que debe tener el perfil del candidato las expone A. C.:

"Para elegir al candidato es necesario que tenga una formación académica, eso se está exigiendo ahora. Por otro lado también sería de ver cuál es el comportamiento de tal persona, el respeto y todo eso. Tercero sería también ver si la persona algún rato fue dirigente, o hizo algo para la comunidad o no hizo" (Entrevista A.C.).

El tema de la formación académica constituye un debate interno ya que quienes han tenido acceso a la educación superior han sido pocos, siendo ellos quienes han tenido mayor acceso a las candidaturas y los otros puestos. Por otra parte, la formación académica aproxima más efectivamente a los candidatos (en lo que se refiere a la acumulación de capital cultural) con los políticos tradicionales, que ocupan los puestos burocráticos y con los cuales se va a convivir políticamente. Desde esta perspectiva, elegir un candidato con formación académica forma parte de una estrategia de apropiación política de los espacios en los cuales se va a actuar, significa ganar espacio con una persona que maneja un lenguaje político y técnico apropiado para competir de igual a igual con los demás y muchas veces superar.

Es justamente la persistencia de la reelección y la ocupación prolongada de los puestos públicos de parte de ciertos dirigentes, lo que contribuye a la formación de una dirigencia delimitada conformada por los dirigentes que se han dado a conocer en sus puestos públicos. Esta concentración del poder en un grupo de dirigentes produce un efecto de distanciamiento con las bases del movimiento que no llegan a tener un contacto efectivo con la dirigencia; poniendo en práctica acciones de un tipo de representación donde salen a la vista simplemente ciertas figuras políticas de trascendencia debido al tiempo de gestión o al nivel de gestión que se ha realizado. Con esto hay que señalar también, que los dirigentes que han realizado una función positiva y se han retirado de sus puestos para dar paso a otros concentran un prestigio en torno a la honestidad y la coherencia con los principios del movimiento que proviene justamente de las bases.

Dentro de este aspecto cabe señalar que la selección de dignidades y su posterior puesta a prueba en las elecciones consiste en un proceso donde el poder opera en una sola dirección; muchos de los dirigentes que han adquirido un capital político fuerte, establecen pactos en función del beneficio de sus organizaciones; en ese sentido, la elección de 
autoridades del MICC y Pachakutik, ha consistido en la configuración política de acuerdo a la fuerza política que detenta cada organización. En el octavo Congreso del MICC en Poaló, se pudo ver como antes del momento de las elecciones un grupo de dirigentes salía del establecimiento y realizaba pactos (como en el Congreso Nacional) con el objeto de asegurar el triunfo de los candidatos que convenían para su organización. En ese sentido la toma de decisiones también puede verse condicionada por aspectos extra-democráticos donde el poder se aplica entre los dirigentes con mayor capital político acumulado.

\section{Reflexiones finales}

Se puede afirmar que el MICC no ha tenido un proceso completamente autónomo sino que en este proceso se han involucrado lógicas de "acompañamiento" las cuales han delineado la ruta a seguir del movimiento; sin embargo, esta perspectiva de los agentes externos, en el caso de la Iglesia por ejemplo, atiende a una acción más bien práctica y no ideológica. Lo que se evidencia es que las reivindicaciones y las demandas planteadas por el movimiento responden a realidades cercanas y cotidianas de los actores internos, es decir, del mismo sector indígena y sus instituciones.

Se ha definido que los procesos por los que atraviesa el MICC, han sido similares a la dinámica del movimiento indígena a nivel nacional, de esta manera se identifican tres episodios. Un primer momento, se ha identificado con el proceso de concentración del capital político, en el cual las demandas han estado direccionadas hacia la reivindicación de los derechos. Un segundo momento, ha sido identificado a partir de la década del noventa, específicamente luego del levantamiento de 1990 (Inti Raymi), a partir del cual inicia un proceso de consolidación del capital político acumulado durante la década anterior, emprendiendo acciones de protesta directa frente al sistema, encontrando en los gobiernos de turno (manifestados en las gobernaciones y demás instancias de ámbito gubernamental a nivel local), el principal adversario. Este momento estaría caracterizado por levantamientos frecuentes a lo largo de toda la década manteniéndose hasta inicios de la siguiente década. El tercer momento, se identifica con la participación electoral a partir de la fundación del partido Pachakutik y consiste en un proceso de utilización del capital político acumulado durante el primer momento de reivindicación y consolidado en la protesta; se ha visto que este tercer momento ha tenido logros efectivos a nivel local donde se ha obtenido el acceso a varios puestos de administración.

Se ha visto que en ciertas ocasiones algunas prácticas contribuyen para "monopolizar el poder" al interior del MICC, lo cual ha demostrado que en cierta medida fracturan la comunicación con las bases. La conformación de una élite política al interior del movimiento ha producido un cierto desentendimiento de las comunidades con la cúpula; las decisiones no se las toma "comunalmente", sino que frente a ciertos aspectos se decide exclusivamente a cierto nivel. Sin embargo, en el caso de la administración de los gobiernos locales, se ve que la "rendición de cuentas" se produce frecuentemente; además el acceso a los gobiernos locales de parte de indígenas es completamente abierto; en cierta medida, ha contribuido para la eliminación del racismo. 


\section{Bibliografía}

Almeida, José (et. al.), 1993, Sismo étnico en el Ecuador; Quito, CEDIME / ABYA - YALA.

Almeida, José (et. al.), 1994, "Identidad, comunicación y modernidad en América Latina”, en: Herlinghaus, Hermann Walter, Monika (eds.), Posmodernidad en la periferia: Enfoques latinoamericanos de la nueva teoría cultural, Langer Verlag, Berlín.

Bourdieu, Pierre, 1997, Razones prácticas, Anagrama, Barcelona.

1991, El sentido práctico, Taurus, Madrid.

Bretón, Víctor, 2002, Comentarios a lo comentado: reflexiones a Tenor de los comentarios de Pablo Ospina; Ecuador Debate \# 55; CAAP, Quito.

2001, Cooperación al desarrollo y demandas étnicas en los Andes ecuatorianos, FLACSO / Universitat de Lleida / GIEDEM, Quito.

2000, El "desarrollo comunitario" como modelo de intervención en el medio rural, Quito, CAAP.

Dávila, Gloria (coord.), 1998, "Diagnóstico comunicacional de las organizaciones campesinas de Cotopaxi”, en: Prieto, Daniel, Tres experiencias de diagnóstico de comunicación; CIESPAL, Quito.

Gangotena, Francisco y Amílcar, Albán, 1982, La UNOCAPAC y el DRI Salcedo, Ecuador Debate \# 61; CAAP, Quito.

Gramsci, Antonio, 1988, Antología, Siglo Veintiuno, México.

Guerrero, Fernando y Pablo Ospina, 2003, El poder de la comunidad. Movimiento indígena y ajuste estructural en los andes ecuatorianos, CLACSO, Buenos Aires.

Ibarra, Hernán y Pablo Ospina, 1994, Cambios agrarios y conflictos de tierra en Cotopaxi, FEPP, Quito.

Krainer, Anita, 1996, Educación intercultural bilingüe en el Ecuador, Abya-Yala, Quito.

Larrea, Ana María, 2005, "Los desafíos del proceso de democratización en Cotopaxi”, en: Unda, Mario (org.), Experiencias en gestión y desarrollo local, CIUDAD / EED/EZE / UASB, Quito.

Martínez, Carlos y Bolívar Burbano, 1994, La educación como identificación cultural y la experiencia de educación indígena en Cotopaxi, Abya-Yala / SEIC, Quito.

Martínez, Luciano, 2002, Desarrollo rural y pueblos indigenas: las limitaciones de la praxis estatal y de las ONG en el caso ecuatoriano, Ecuador Debate \#55; CAAP, Quito.

MICC, 2002, Estatuto y Reglamento Interno, Latacunga / Quito, MICC.

Ortiz Crespo, Gonzalo, 1992, "El problema indígena y el gobierno", en: Almeida, Ileana et. al., Indios. Una reflexión sobre el levantamiento indígena de 1990, ILDIS / Abya-Yala, Quito.

Ospina, Pablo, 2002, Crítica Bibliográfica al libro Cooperación al desarrollo y demandas étnicas en los Andes ecuatorianos, Ecuador Debate \# 55, CAAP, Quito.

Ospina, Pablo (coord.), 2006, En las fisuras del poder. Movimiento indigena, Cambio social y gobiernos locales, IEE / CLACSO, Quito.

Reyes, Eloy Alfaro, 2005, Quién pone el cascabel al gato: sistematización de la convención ambiental "cotopaxi en minga", Quito, IEE, Disponible al 9 de Mayo de 2007 en: www.grupochorlavi.org/ gobernanzaambiental/Completos/Cotopaxi.pdf .

Rosero, Fernando, 1990, Levantamiento Indígena: tierra y precios, CEDIS, Quito,

Santana, Roberto, 2004, Cuando las élites dirigentes giran en redondo: el caso de los liderazgos indígenas en Ecuador, Ecuador Debate \# 61; CAAP, Quito.

1983, Campesinado indígena y el desafio de la modernización, CAAP, Quito.

Tibán, Lourdes et. al., 2003, Movimiento Indígena y Campesino de Cotopaxi (MICC). Cotopaxi Markamanta Runakunapak Jatun Kuyurimuy. Historia y proceso organizativo, Latacunga, MICC.

Tibán, Lourdes, 2004, El sistema de representación y la participación política del pueblo kichwa de Cotopaxi; Quito, Tesis para la obtención de la maestría en Ciencias Sociales con mención en Asuntos Étnicos; FLACSO, Quito.

Touraine, Alain, 1984, El regreso del actor, EUDEBA, Buenos Aires.

Varela, Roberto, 2005, Cultura y poder, Anthropos/Universidad Autónoma Metropolitana, Barcelona.

Weismantel, Mary J., 1994, Alimentación, género y pobreza en los Andes ecuatorianos, Abya - Yala, Quito. 


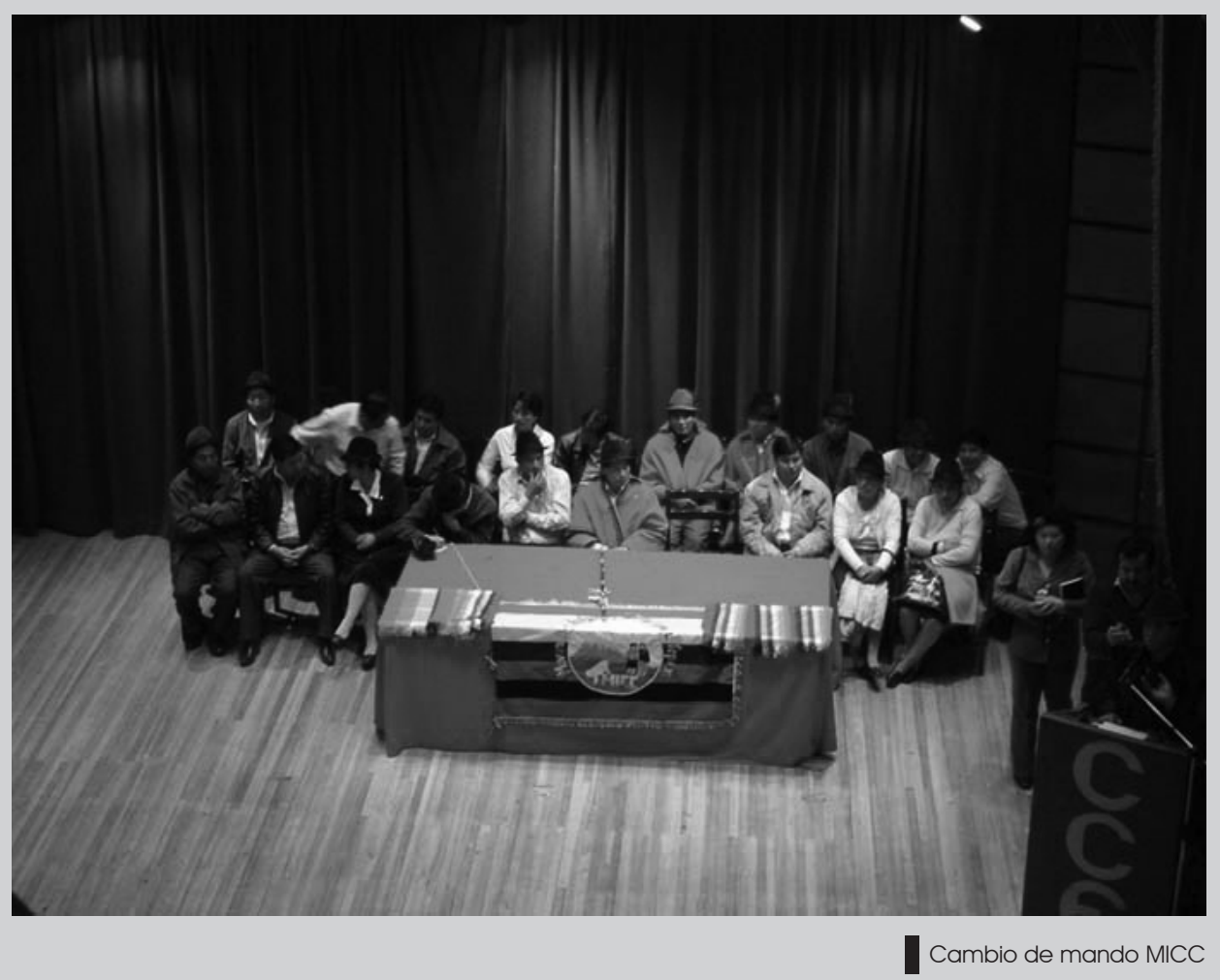




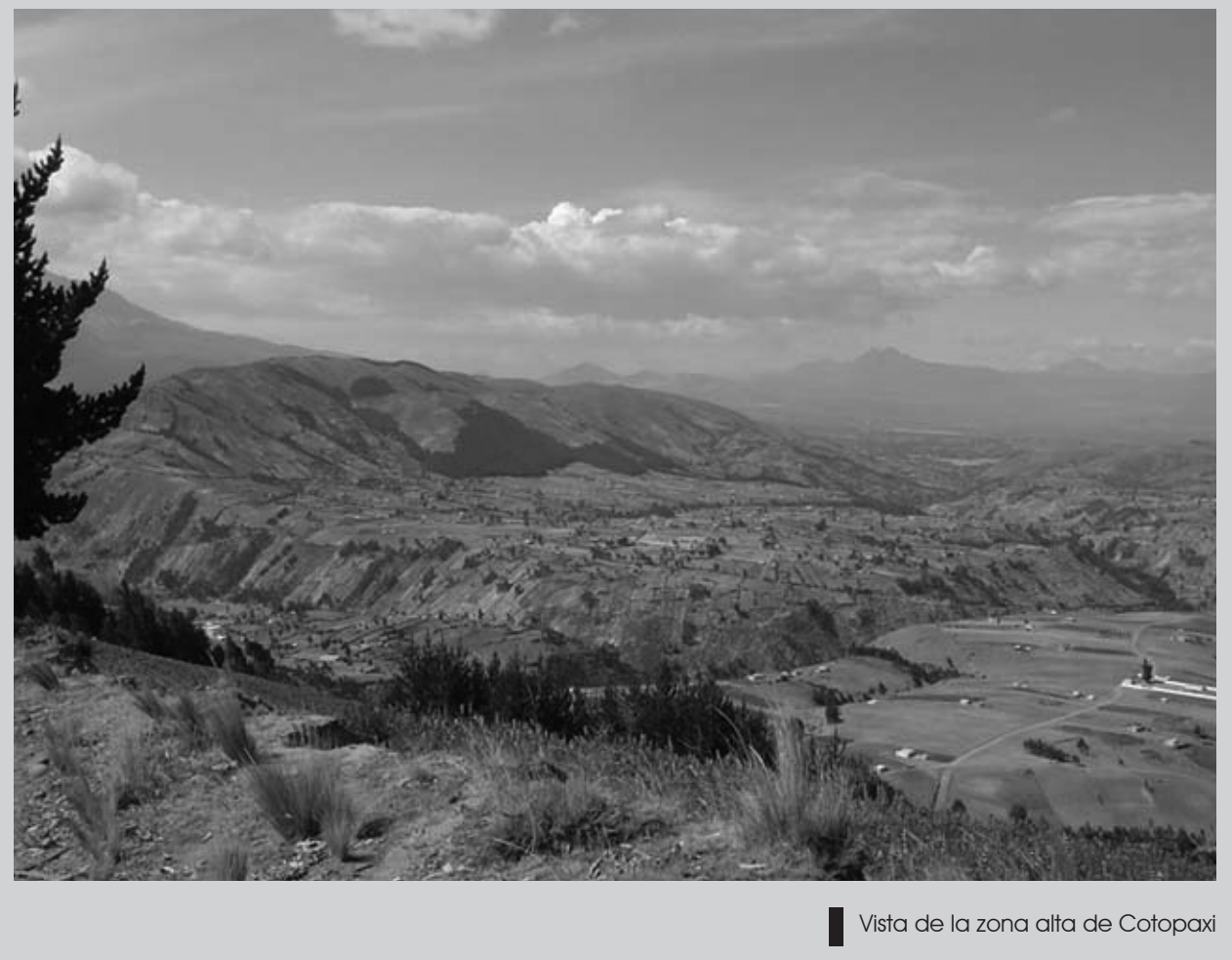

